\title{
COSMOLOGICAL INFORMATION FROM NEW TYPES OF RADIO OBSERVATIONS
}

\author{
William C。Saslaw \\ University of Virginia, and National Radio Astronomy Observa- \\ tory*, Charlottesville, and Institute of Astronomy, Cambridge, \\ England.
}

To have squeezed the Universe into a ball

To roll it toward some overwhelming question.

T. S. Eliot

So far, we have mainly discussed what might be called classical methods for investigating cosmological models using radio observations. These mostly involve the number counts as a function of measured flux, angular diameters, and spatial distribution of radio sources at cosmological distances, as well as the measurements of the microwave background. In this review, I have been asked to give a brief description of some new, or non-classical, measurements that might be made. It seems that such measurements fall into one of three categories: Observations which are being made now and which may bear fruit in the next several years, observations which may be possible in the intermediate future several decades from now, and observations which may be possible in the far future - if ever. Naturally I'll try to concentrate on the first two categories since they will be of more interest to most living astronomers during their working lifetimes. Also, since time is short and it is very difficult to predict the future, I'm afraid that this review will be somewhat impressionistic rather than exhaustive. I hope people will describe additional methods during the discussion.

\section{OBSERVATIONS POSSIBLE NOW}

There are a number of new types of observations which have been made recently and which will be extended in the next several years to give cosmological information. One of the most rapidly developing methods is the radio analogue of the optical magnitude-redshift-diagram.

* The National Radio Astronomy Observatory is operated by Associated Universities, Inc. Under contract with the National Science Foundation. 
This is being pursued by Fisher and Tully at NRAO and Marseille. The basic idea is to measure the $21 \mathrm{~cm}$. redshifts of a large number of spiral galaxies - which typically requires only several minutes per redshift with a large low noise telescope - and then to find an indicator of each source's intrinsic $21 \mathrm{~cm}$. radio magnitude. Measuring the apparent $21 \mathrm{~cm}$. magnitude would then, in principle, enable one to plot the standard Hubble diagram and determine $\mathrm{H}_{\mathrm{O}}$ and possibly $\mathrm{q}_{\mathrm{O}}$ (without the important evolutionary radio corrections, of course). Alternatively, if the $21 \mathrm{~cm}$. measurement provides an indicator of a sources intrinsic optical luminosity, its apparent optical magnitude could be used.

The chief problem with this method therefore is finding a reliable indicator of the source's intrinsic luminosity. There are several possibilities. It may be that for galaxies in a narrow morphological class, the intrinsic $21 \mathrm{~cm}$. Iuminosity can be shown to depend mainly on mass. To do this one would examine galaxies whose distance is known independently (e.g. members of the Virgo Cluster) and whose mass can be found from the width of the $21 \mathrm{~cm}$. profile. Since these results would also depend on the size and orientation of the galaxy, it is necessary to measure a large statistically homogeneous sample. An exploratory start in this direction has been made by Fisher and Tully (1975) using 241 dwarf galaxies catalogued by the David Dunlap Observatory. They have found some weak evidence for a slightly simpler relation: a dependence of the hydrogen $\mathrm{M}_{\mathrm{H}} / \mathrm{L}$ ratio (which does not depend on distance) on the absolute photographic magnitude of the irregular dwarf DDO galaxies. Here the hydrogen mass is determined directly from the $21 \mathrm{~cm}$. flux density corrected for beam dilution, and $L$ is the intrinsic photographic luminosity. And they have also found stronger evidence for a relation between a measure of the total mass of a galaxy and its absolute photographic magnitude. The total mass measure of the galaxy is taken to be proportional to (distance). (Holmberg angular diameter).(full line width at half intensity) $)^{2}$ ( $\operatorname{cosec}^{2}$ inclination). Thus if these two relations were to hold, it would be possible to measure the distance independently of the redshift and have another determination of $\mathrm{H}_{\mathrm{O}}$. Another possibility is that the shape of the $21 \mathrm{~cm}$. Iine profile is itself related to the intrinsic luminosity of the galaxy for a given morphological type. This whole area is just beginning to be understood as large statistical samples accumulate and the absolute calibrations of the telescope are determined. Moreover, very little has been done in deriving detailed theoretical models of evolving $21 \mathrm{~cm}$. Iine profiles to be expected from different types of galaxies. In future such studies will prove very useful.

Another contribution which radio observations make to cosmology is through measurements of the total mass density, $\rho_{\circ}$, of the Universe. For a Friedman universe dominated by a non-relativistic fluid, the ratio of the actual density to the critical density, $\rho_{c}$, needed to close the universe is just $2 \mathrm{q}_{O}$, when the cosmological constant is zero. If the energy density is dominated by a relativistic fluid, then $\rho_{O} / \rho_{c}=q_{O}$. There have been many attempts to determine the amount of intergalactic neutral hydrogen in $\rho_{0}$. Most recently Lang (1976) has searched for 
neutral hydrogen in the directions of four radio galaxies and found upper limits $\rho_{\mathrm{H}}<\rho_{\mathrm{c}}$ in all cases. Relativistic ionized hydrogen in clusters has also been searched for by its effect on the microwave background. Here the idea is to detect any diminution of the background radiation at microwave frequencies in clusters caused by its inverse Compton scattering to higher frequencies by local relativistic electrons. Both Partridge and Gull (described at this meeting) have looked at $9 \mathrm{~mm}$. and $3 \mathrm{~cm}$. respectively in several rich clusters. So far there is no strong indication of this effect at a sensitivity of about $10^{-3} \mathrm{~K}$. Although the interpretation of these results is model dependent, it suggests that the mass of ionized gas is considerably less than the "virial mass" and thus much less than $\rho_{\mathrm{c}}$.

The mass density of matter in galaxies may also be found by measuring redshifts for a large complete sample of galaxies. This is an extension of the old virial theorem argument for groups to the statistics of velocity distributions in the sample. The galaxies in this sample need not be divided into groups. These statistical virial measures are based on the departure of the velocity distribution from a smooth Hubble flow (Peebles, 1976; Fall, 1976; Sargent \& Turner, submitted to Ap. J.). other things being equal, larger correlated departures from a smooth Hubble flow at present indicate a greater density of clumped matter. Since the dispersion around the Hubble flow may be quite small, it is necessary to have accurate redshifts of the galaxies, and this can be done best by measuring the $21 \mathrm{~cm}$. emission line. Thuan and Knapp are doing this for a complete sample of about 1100 galaxies in the Zwicky catalogue with $\mathrm{M}_{\mathrm{pg}} \geqslant 14,|\mathrm{~b}|>40^{\circ}$ and $\delta>0^{\circ}$. Some 90 per cent of these galaxies are spirals and their HI radial velocities can be measured on the NRAO 300-foot to an accuracy of about $15 \mathrm{~km} \mathrm{~s}^{-1}$ in about five minutes apiece.

While this will provide a major important estimate of the mass density, this technique also has its difficulties. One is that it does not include any matter such as hot gas, radiation, or neutrinos which is distributed uniformly throughout the Universe. Another is that it assumes a cold initial Hubble flow with no random or correlated velocities. Although the process of galaxy formation remains a mystery, it is quite likely that the initial perturbations from which they arose also included longer wavelength perturbations which started them off with a clustered (i.e. non-random) distribution. If they had a positive initial correlation, this technique would lead to an overestimate of $\rho_{\mathrm{o}} / \rho_{\mathrm{c}}$, while if they were anti-correlated initially this would underestimate $\rho_{o} / \rho_{c}$.

Next we turn from observations of parameters of Friedman models to other questions of cosmology. One of the most basic is whether the fundamental constants of laboratory physics have changed during the evolution of the Universe. The best known of these is Dirac's speculation that the constant of gravitation may decrease as the Universe ages so as to preserve the equality between the dimensionless ratio of electric and gravitational forces and the dimensionless age of the universe. 
However the a priori question of whether these quantities change is a broader one, to be settled observationally unless one can show that such constancy is required for our conceptual structure of physics to be internally consistent.

Recently a radio observation has put strong constraints on temporal changes of atomic and nuclear physical constants (Wolfe, et al. 1976). The quasar AO $0235+164$ has both hydrogen hyperfine absorption lines and MgII fine structure lines at the same redshift; $z=0.5239 \pm .0001$. By comparing the terms responsible for the hyperfine splitting of the hydrogen ground state with the fine structure splitting of the MgII doublet, one finds that the product $\alpha^{2} \mathrm{~g}_{\mathrm{p}} \mathrm{m} / \mathrm{M}$ has changed by less than two parts in $10^{14}$ per year over the roughly 1010 years which have elapsed since the radiation was emitted. Here $\alpha$ is the fine-structure constant, $g_{p}$ is the nuclear $\mathrm{g}$ factor for the proton, and $\mathrm{m} / \mathrm{M}$ is the electron/proton mass ratio. Presumably this limit also applies approximately to each of the factors of the product unless there is some fortuitous cancellation. Discovery of more examples at higher redshifts would improve this limit and also check the expectation that the effect is isotropic.

Another constraint on changes of a fundamental constant may be provided by the microwave background. Noerdlinger (1973) has pointed out that if Planck's constant is taken to be potentially variable, the black-body spectrum of the radiation requires the fractional change of hc with redshift $\mathrm{z}$ to be less than three parts in $10^{4}$ since decoupling. This is derived by noting that the temperature of the background derived from the Rayleigh-Jeans part of the spectrum, which does not depend on $\mathrm{h}$, is the same (to within about 30 per cent) as the temperature derived from the turnover of the spectrum, which does depend on $h$. The main question here, however, is whether one would really expect $h$ to change in principle, since it is a dimensional quantity and its value is really just a convention. Much as the speed of light is a conventional conversion factor between length and time, so h would be a conversion factor between, say, energy and frequency.

Measurements at millimeter wavelengths of the polarization of the microwave background could provide especially interesting information about any early anisotropy of the universe (Rees, 1968). Anisotropic radiation becomes linearly polarized when it is scattered by free electrons, so if the universe expanded anisotropically, meaning that the radiation had a different temperature along different axes of expansion, part of the microwave background would be polarized. For a given amount of anisotropy, the fractional polarization depends mainly on the ionization history of the gas during and after decoupling. Under the most favourable conditions, if the present density. of intergalactic gas is greater than $10^{-5} \mathrm{~cm}^{-3}$ and it was reionized at $\mathrm{z} z 7$, the fractional polarization can be several times the fractional temperature anisotropy. Inhomogeneities in the matter distribution will also produce some polarization, especially if they have large proper motion relative to an observer and therefore scatter anisotropically. At present these effects have not been detected at the 1 per cent level, but more sensitive experiments should be possible within the next few years. 


\section{OBSERVATIONS WHICH MAY BE POSSIBLE IN THE INTERMEDIATE FUTURE.}

If we look beyond the near future, the only safe prediction, of course, is that any predictions we make will be covered by surprises. Nevertheless, people have made several suggestions for new observations.

There is an interesting area which may show the effects of the microwave background on radio galaxies. Once this is calibrated and understood, the argument can be inverted to give a rough independent estimate of the redshift of a distant radio source without knowing its optical spectrum. The idea is to measure the diffuse optical emission in the radio lobes of radio galaxies which is produced by inverse Compton scattering of synchrotron electrons on the black body background. Recently, P. Crane, J. A. Tyson, and I have found some possible evidence for diffuse optical emission in the radio lobes of $3 \mathrm{C} 390.3$ and $3 \mathrm{C} 285$. Its intensity is between about 2 per cent and 10 per cent of the night sky background. We do not know yet whether this optical emission is the visible extension of the radio synchrotron or if it is inverse Compton scattering of the microwave background. Either can be made consistent with the present data, but it should be possible to distinguish between them in future. By measuring the polarization and spectrum, one can estimate the fraction of optical radiation which could be caused by inverse Compton scattering. The ratio of optical inverse Compton to radio synchrotron emission from a small volume depends only on the electron spectrum, the magnetic field, and the redshift of the source (through the $z$ dependence of the energy of the microwave background). One can estimate the electron spectrum if one is willing to make the usual extrapolation from the spectral index of the observed radio synchrotron emission. The magnetic field can be found independently from the angular size if the synchrotron emission is self-absorbed. Thus the redshift can be estimated roughly since the ratio of inverse Compton to synchrotron radiation varies as $(1+z)^{3+\alpha}$ where $\alpha$ is the spectral index of the synchrotron radiation - typically . $5 \leqslant \alpha \leqslant 1$. Although we would not expect this to give very accurate redshift information, it might eventually distinguish between redshifts of, say, 0.1 and $I$. However, until we understand the physics of radio sources better, it is more reasonable to invert the argument for cases where we know the redshift of the optical galaxy connected with the source. Then this procedure gives an estimate of the magnetic field in the source and constrains models further. Incidentally, the optical synchrotron part of the emission sets very short lifetimes on the optically radiating electrons. This requires them to be generated close to the region where they radiate. The main observational development we need for this is a high resolution device to measure polarization and colors (if not spectra) at less than 1 per cent of the night sky background.

So far this discussion has mainly been concerned with short wavelengths, i.e. $\lambda \leqslant 2 l \mathrm{~cm}$. Observations at longer wavelengths could also provide interesting cosmological information. One such observation has been suggested by Sunyaev and Zeldovich (1974). This is to search for the redshifted $21 \mathrm{~cm}$. radiation produced by protogalaxies. If galaxies 
formed according to fairly conventional ideas, most of their mass was once in the form of neutral hydrogen. If the velocity dispersion in a protocluster of this gas was $210^{3} \mathrm{~km} \mathrm{~s}^{-1}$, it would lead to a $21 \mathrm{~cm}$. Iine of width $\Delta v / v \leq 10^{-3}$. This would be redshifted to a wavelength of a couple meters if galaxies formed at redshifts around 5-10. The flux density in such a line would be between about one and a few millijanskys, depending on whether $q_{0}$ was of order unity or of order 0.1 . The detection of this radiation depends critically on it being in a very narrow line so it can be distinguished from the background. This in turn depends on the hydrogen in protoclusters existing in a neutral state only during a very narrow range of redshift. If there are many galaxies along the line of sight radiating over a large range of redshift, the line will be correspondingly smeared out and difficult to find. Thus this type of observation would give information on the manner in which galaxies and clusters of galaxies formed, and on the relevant cosmological boundary conditions.

Similarly, the time may come when high resolution spectra of distortions of the microwave background reveal as much about the history of matter in the early Universe as the spectra of ionized gases revealed about the inside of the atom. There has been a large amount of theoretical work on this problem during the last decade, and it is discussed in IAU Symposium No.63 (Longair, ed. 1975) as well as in this symposium. So I won't describe it further here.

A quite different type of observation which may eventually become practical is to measure the dispersion in a pulse caused by ionized intergalactic gas (Haddock \& Sciama, 1965). If the pulse comes from a distant source, the time delay in its reception at low and high frequencies is a function of $\mathrm{q}_{\mathrm{O}}, \mathrm{H}_{\mathrm{O}}, \mathrm{z}$, and the electron density between the source and the observer. If $\mathrm{z}$ is measured independently, then observations of many sources can provide a best fit to $\mathrm{q}_{\mathrm{O}}, \mathrm{H}_{\mathrm{O}}$, and $\mathrm{n}_{\mathrm{e}}$. Here one must also determine the part of the dispersion caused by electrons in clouds surrounding the source. The ideal source would be a distant pulsar. But this would require a great increase in our present sensitivity to pulsating signals. It is also conceivable that pulses are produced by the mechanisms which generate radio emission in galactic nuclei and extended radiogalaxies.

\section{OBSERVATIONS FOR THE FAR FUTURE - IF EVER.}

Taken at face value, one of the simplest ways to find $q_{0}$ is to measure it directly. One can readily show that the cosmological expansion changes the redshift of a distant source as seen by a local observer at the rate

$$
\frac{d z}{d t_{0}}=H_{0}(I+z)\left[I-\left(I+2 q_{0} z\right)^{\frac{1}{2}}\right]
$$

for any redshift, $z$, in a matter dominated Friedman universe. This 
agrees with our expectation that if expansion does not accelerate, i.e. if $\mathrm{q}_{O}=0$, the relative velocity of the source remains constant. For small $\mathrm{z}$, the fractional change in redshift becomes d lnz/dt $\mathrm{O}=-\mathrm{H}_{\mathrm{O}} \mathrm{q}_{\mathrm{O}}$. Thus by measuring $\mathrm{z}$ to one part in about $10^{8}$ at intervals of about 100 years, one could hope to determine $q_{0}$ directly. At present radio redshift measurements are one to two orders of magnitude more accurate than optical redshifts. Davis (1975) has suggested applying this method to the $21 \mathrm{~cm}$. absorption line of $3 \mathrm{C} 286$ ( $\mathrm{z} \approx 0.8$ ) which has a half-width of $25 \mathrm{KHz}$. The absorption redshift can be determined to one part in $10^{6}$. With this accuracy we would have to wait about ten thousand years for second epoch measurements, and even astronomers are not that patient. However a factor of about 10 improvement in accuracy is possible now with narrower lines, a further improvement of order unity in waiting time is possible if higher redshift $(\mathrm{z} z 3)$ lines are discovered, and if another factor of about 10 is granted for unexpected developments, then it is conceivable that this method could give a result over the next few decades. It might even become possible to apply it optically with new types of detectors.

To use this method practically, it would be necessary to apply it to a large number of sources. The reason is that the cosmological deceleration is only one cause of change in the source's redshift. There will also be changes in the internal structure of the source, and changes due to the local acceleration of the source by nearby sources. All these effects will have to be averaged out to find the residual cosmological deceleration.

There is another type of experiment which, if it can be done, would not only occur in future, but would tell us about the future of the Universe. This involves the question of whether it is possible to distinguish between Maxwell electrodynamics and Wheeler-Feynman absorber theory. They are equivalent if the Universe absorbs radiation completely along the future light cone, but not if the Universe is an incomplete absorber. Thus an experiment performed now would depend upon the advanced radiation produced along our future light cone. This would contradict our usual notions of causality. The first experiment to attempt to distinguish between the two theories was Partridge's (1973). He measured the power input to a microwave source as it alternatively radiated into free space (where very little energy was absorbed locally) and then into an efficient local absorber. The power input to the source remained constant to one part in $10^{8}$ as the output was switched between these two regions. However it has been pointed out (Pegg, 1973; Heron and Pegg, 1974; Davies, 1975; Pegg, 1975) that not all forms of absorber theory can be distinguished by this experiment, and the null result may have been independent of the cosmological boundary conditions. One might also be able to account for any observed power difference using Maxwell's theory in terms of ordinary retarded radiation from background sources. At present, this subject is in a rather controversial state and people are attempting to devise more powerful experiments. 
Finally, some of my colleagues have suggested that the easiest way to find out about the Universe would be to wait until we are discovered by a more advanced civilization. Then we could ask them. But even if this turns out to be true, surely it is much more fun to try to roll the Universe toward these questions ourselves.

Acknowledgments

Part of this review was written at the Aspen Center for Physics during the summer of 1976.

\section{References}

Davies, P.C.W.: 1975, J. Phys. A. Math. Nucl. Gen. 8, 272.

Davis, M.M.: 1975, Bulletin AAS 7, 236.

Fall, S.M.: 1976, Montly Notices Roy. Astron. Soc. 176, 181.

Fisher, J.R. and Tully, R.B.: 1975, Astron. Astrophys. 44, 151.

Haddock, F.T. and Sciama, D.W.: 1965, Phys. Rev. Letters 14, 1007.

Heron, M.C. and Pegg, D.T.: 1974, J. Phys. A. Math. Nucl. Gen. 7, 1965.

Lang, K.R.: 1976, Astrophys. J. Letters 206, L91.

Longair, M.S.: 1975, IAU Symp. 63.

Noerdlinger, P.D.: 1973, Phys. Rev. Letters 30, 761.

Partridge, R.B.: 1973, Nature 244, 263.

Peebles, P.J.E.: 1976, Astrophys. J. Letters 205, L109.

Pegg, D.T.: 1973, Nature Phys. Sci. 246, 40.

Pegg, D.T.: 1975, J. Phys. A. Math. Nucl. Gen. 8, L60.

Rees, M.J.: 1968, Astrophys. J. Letters 153, Ll.

Sargent, W.L.W. and Turner, E.: 1976, Astrophys. J. submitted.

Sunyaev, R.A. and Zeldovich, Ya.B.: 1974, Monthly Notices Roy. Astron. Soc. 171,375 .

Wolfe, A.M., Brown, R.L. and Roberts, M.S.: 1976, Phys. Rev. Letters in 


\section{DISCUSSION}

E.M. Burbidge: You have said nothing about all of the things we would hope to find, in the intermediate future, with the "Large Space Telescope".

Setti: I have made computations of the expected optical fluxes from radio components due to inverse Compton of the relativistic electrons against the $2.7 \mathrm{~K}$ background photons. For instance, in the case of equipartition between particle and field energies (protons/electrons $=1$ ) and for $q_{0}=1$ and a spectral index $\alpha=-1$, one finds

$$
m_{B} \approx 31.8-1.25 \log \left[\frac{S_{178}(\mathrm{Jy})(\mathrm{Hz})^{4}}{\left(\Delta \phi^{11}\right) \mathrm{z}(\mathrm{Ho} / 50)}\right]\left(\frac{\mathrm{mag}}{(\operatorname{arc~sec})^{2}}\right)
$$

where $\Delta \phi^{11}$ is the diameter of the radio component in arc sec and $z$ is the redshift. So that in some cases one may expect $\mathrm{m}_{\mathrm{B}} \approx 29 \mathrm{mag} /(\mathrm{arc} \mathrm{sec})^{2}$. Bright and closeby sources, like Cygnus A, would of course provide a stronger case. It is perhaps unfortunate that the location of Cygnus $A$ in the sky may make it difficult to test the existence of any weak optical emission.

McCrea: The work of $w$. Baum should not be overlooked in any discussion of possible changes in the constants of physics. 